\begin{tabular}{|c|c|}
\hline $\begin{array}{l}\text { MINING AND METALLURGY INSTITUTE BOR } \\
\text { UDK: } 622\end{array}$ & $\begin{array}{l}\text { ISSN: 2334-8836 (Štampano izdanje) } \\
\text { ISSN: 2406-1395 (Online) }\end{array}$ \\
\hline UDK: $620.193: 546.76 / .11(045)=111$ & doi:10.5937/MMEB1503145J \\
\hline
\end{tabular}

\title{
MECHANISMS OF HYDROGEN EVOLUTION ON CHROMIUM ${ }^{* *}$
}

\begin{abstract}
In this work, the mechanisms of hydrogen evolution on the metal chromium in a solution of $0.1 \mathrm{M}$ $\mathrm{Na}_{2} \mathrm{SO}_{4}+\mathrm{H}_{2} \mathrm{SO}_{4}, \mathrm{pH} 1$ to $\mathrm{pH} 7$ were analysed. In the $\mathrm{pH}<3$ range, hydrogen is evolved according to three different mechanisms, depending on the experimental conditions: electrochemical evolution of $\mathrm{H}_{2}$ by reaction of $\mathrm{H}^{+}$ions according to the Volmer-Heyrovsky mechanism, on the bare chromium surface, during chemical dissolution of chromium by the Kolotyrkin mechanism, which does not depend on the electrode potential, and electrochemical hydrogen evolution by the Volmer-Heyrovsky mechanism, but on the passive chromium. At pH values greater than 3, the fourth mechanism of hydrogen evolution is observed, that is electrochemical reaction of water molecules on the surface of oxide covered chromium surface.
\end{abstract}

Keywords: chromium, corrosion, chemical dissolution, hydrogen evolution

\section{INTRODUCTION}

In parallel with the electrochemical dissolution of chromium, its chemical dissolution takes place, which does not depend on potential, and is a dominant process of dissolution in some circumstances [1-8]. During chemical dissolution of chromium, the hydrogen evolution occurs, which does not obey the laws of electrochemical kinetics. The chemical evolution of hydrogen can explain the observed processes of corrosion fatigue and stress corrosion of stainless steels in conditions where its electrochemical evolution is impossible. The low current efficiency in galvanic deposition of chromium coatings may be the result of parallel chemical dissolution of chromium during electrodeposition of chromium.

Chromium is in the passive state after immersion in a test solution, with the characteristic corrosion potential $E_{\text {cor.1. }}$. During

cathodic polarization at $-0.900 \mathrm{~V}$ in a few tens of seconds, the passive film dissolves, whereas the chromium electrode is activated. The corrosion potential $E_{\text {cor.2 }}$ is then formed, which corresponds to the active chromium.

\section{EXPERIMENTAL PART}

Samples of metallic chromium were sealed in methacrylate mass, and the sample surface was mechanically polished with abrasive paper, gradually to the fineness 1000 . The samples were then rinsed with distilled water and placed in an electrochemical cell. The test solutions $(0.1 \mathrm{M}$ $\mathrm{Na}_{2} \mathrm{SO}_{4}+\mathrm{H}_{2} \mathrm{SO}_{4}$, pH 1 to $\mathrm{pH}$ 7) were dearated with the use of purified nitrogen.

To perform electrochemical tests, the three-part glass electrochemical cell was

\footnotetext{
*Institute for Chemistry, Technology and Metallurgy, University of Belgrade, Njegoševa 12, Belgrade, Serbia, e-mail: borejegdic@yahoo.com

** This work was supported by the Ministry of Education, Science and Technological Development of the Republic of Serbia, trough the Projects No. 34028 and No. 34016.
} 
used, with water jacket, and with Pt foil as a counter electrode and a saturated calomel electrode (SCE) as a reference electrode. All measured potentials are shown in relation to the SCE.

In some cases, before the measurement, the electrode of chromium was activated by cathodic polarization at $-0.900 \mathrm{~V}$ for $120 \mathrm{~s}$, to remove any surface oxide which can be spontaneously formed on chromium in contact with the air. After $\sim 15$ min of corrosion potential stabilization, polarization curves were recorded potentiodynamically, at sweep rate of $2 \mathrm{mV} \mathrm{s}^{-1}$. Electrochemical measurements were performed using the potentiostate-galvano-state PAR 273.
The preferential orientation of individual grains on the surface of the chromium sample (sample texture) was determined by the electron backscatter diffraction, EBSD.

\section{RESULTS AND DISCUSSION}

Figure 1 shows the inverse polar image of chromium texture obtained by the EBSD method. As can be seen on the coloured scale, the relative frequency of the crystal planes with orientation (111) on the surface of sample is 4.851 times higher than the frequency of sample without texture.

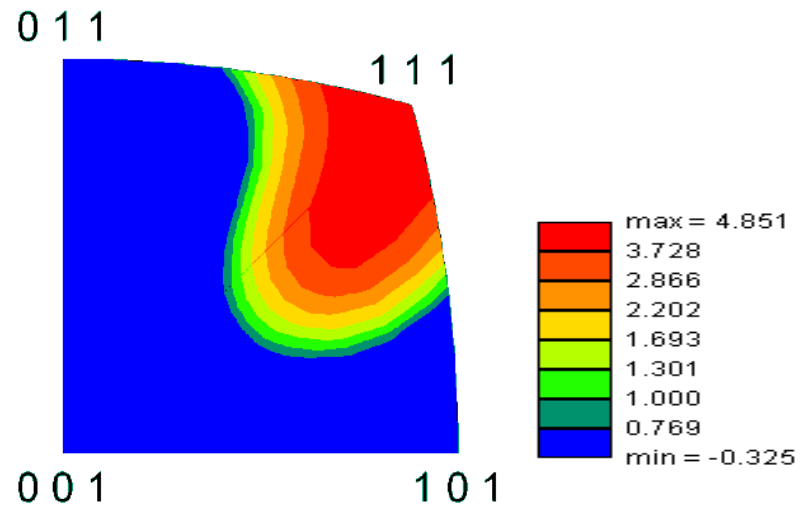

Figure 1 Inverse polar image of chromium texture obtained by the EBSD method. The colored scale indicates the relative frequency of particular crystallographic orientations

Figure 2 shows the cyclic voltammogram of chromium electrode in $0.1 \mathrm{M}$ $\mathrm{Na}_{2} \mathrm{SO}_{4}+\mathrm{H}_{2} \mathrm{SO}_{4}, \mathrm{pH}$ 1. The voltammogram shows the unusual behaviour at reverse change of potential. The recorded current, instead of being always anodic in the potential region between the corrosion potential $E_{\text {cor.2 }}$ and positive limits of the potential (Inset in Figure 2), changes its character twice in this potential region, forming another corrosion potential $E_{\text {cor.1 }}$ and a cathodic peak. This is a cathodic peak of hydrogen evolution on the passive film, but not a peak of oxide reduction. During potential hold in the mentioned potential region, the cathodic current has a stable value, which does not change over time. This indicates that this peak is not a peak of oxide reduction.

The corrosion potential $E_{\text {cor. } 1}$ is formed on the electrode of chromium which is directly immersed in solution. The oxide layer on chromium electrode has been spontaneously formed. $E_{\text {cor.2 }}$ refers to the corrosion potential that was formed on the surface of chromium after cathodic treatment at -0.900 $\mathrm{V}$, for the purpose of oxide film reduction. 


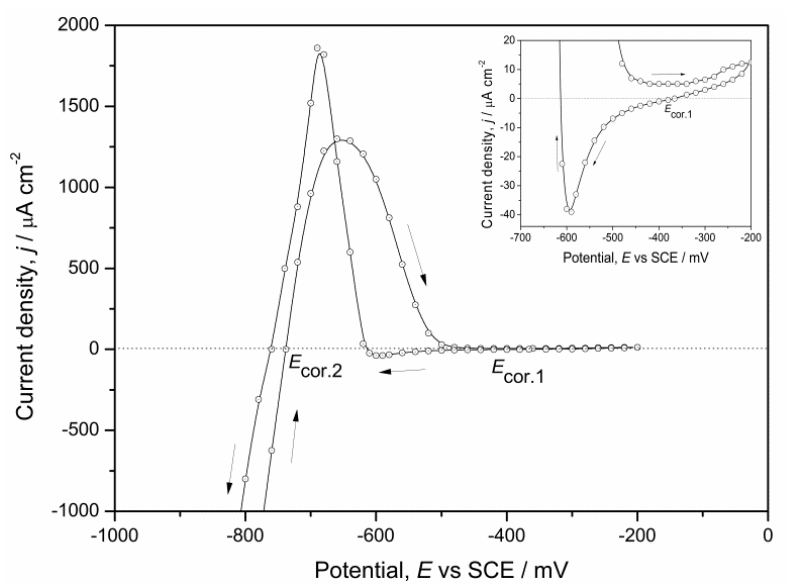

Figure 2 Cyclic voltammogram for chromium electrode after cathodic activation. Inset shows enlarged cathodic peak at reverse potential change. This peak refers to cathodic hydrogen evolution on passivated chromium surface.

A voltammogram example, similar to that in the inset in Figure 2, is shown in Figure 3. Reverse parts of cyclic voltammograms, recorded at different temperatures, express the cathodic hydrogen evolution on the pasivated chromium surface. As it can be seen, cathodic current increases with increasing temperature. However, the position of corrosion potential $E_{\text {cor. } 1}$ and cathodic peak is not changed.

Accordingly, the cathodic current is a result of cathodic hydrogen evolution on the oxide covered chromium surface. The corrosion potential $E_{\text {cor. } 1}$ is controlled by the reaction of cathodic hydrogen evolution and anodic dissolution of chromium through passive film. Hydrogen is evolved on the passivated chromium surface until the potential is reached that corresponds to the potential of oxide dissolution $(\sim-0.580 \mathrm{~V})$. Sample of chromium is then activated, and current rapidly passes from cathodic to anodic region.

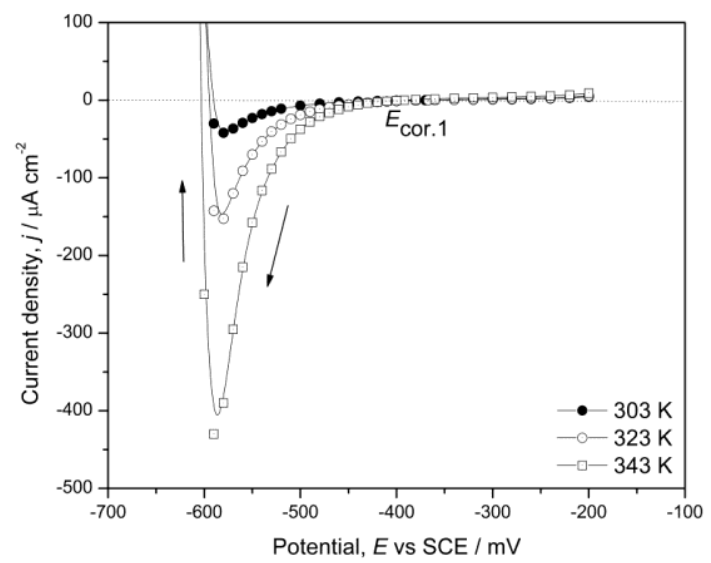

Figure 3 A reverse part of cyclic voltammogram of chromium electrode in solution of $0.1 \mathrm{M} \mathrm{Na}_{2} \mathrm{SO}_{4}+\mathrm{H}_{2} \mathrm{SO}_{4}, \mathrm{pH} \mathrm{1}$, at various temperatures 
At room temperature, the Tafel slopes on the oxide covered chromium electrode, change gradually from the value approximately equal to $0.12 \mathrm{~V} \mathrm{dec}^{-1}$ at $\mathrm{pH} 3$ to the value of $0.06 \mathrm{~V} \mathrm{dec}^{-1}$ to $\mathrm{pH} 1$ [3].

Cathodic and anodic Tafel lines with approximately equal Tafel slopes of about 0.12 $\mathrm{V} \mathrm{dec}{ }^{-1}$ were obtained for chromium electrode, previously cathodically activated in the solutions with a $\mathrm{pH}$ below 3. Typical diagrams for a $\mathrm{Cr}$ in the solutions with a $\mathrm{pH}$ 1 and $\mathrm{pH} 2$ are shown in Figure 4. The intersection of Tafel lines at the corrosion potential $E_{\text {cor.2 }}$ shows that the corrosion potential is determined in terms of the Wagner-Traud model of electrochemical processes for anodic dissolution of chromium and cathodic hydrogen evolution. In parallel with the electrochemical dissolution of chromium the chemical dissolution of chromium occurs according to the Kolotyrkin mechanism [2].

The cathodic hydrogen evolution on the active surface of chromium with a slope of $0.12 \mathrm{~V} \mathrm{dec}^{-1}$ indicates the following reaction:

$$
\mathrm{Cr}+2 \mathrm{H}^{+}+2 \mathrm{e}^{-} \rightarrow \mathrm{Cr}+\mathrm{H}_{2}
$$

This reaction probably occurs by the Volmer-Heyrovsky mechanism.

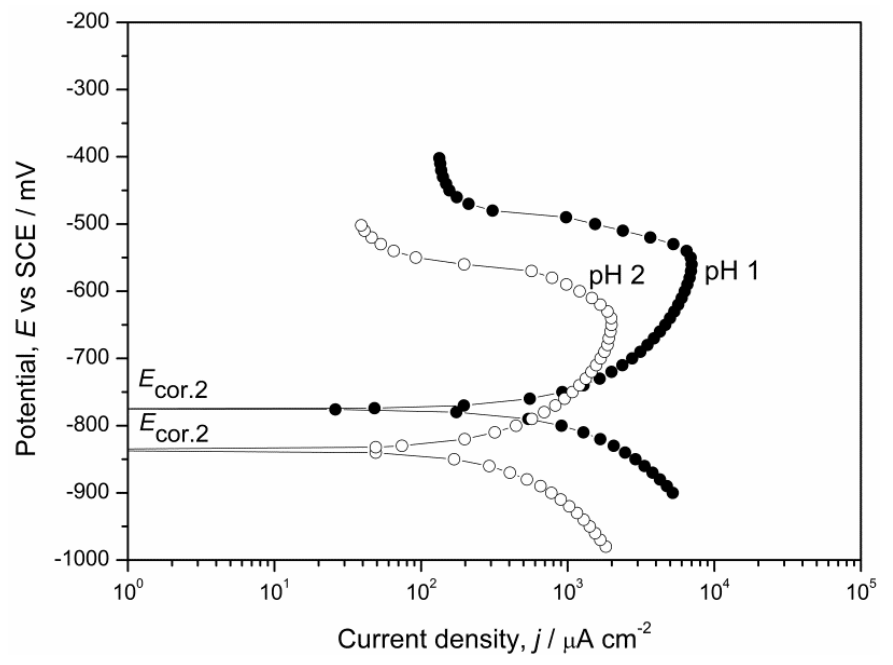

Figure 4 Anodic and cathodic polarization curves for chromium electrode previously cathodically activated, in dependence on the $\mathrm{pH}$ value of solution

Corrosion potential $E_{\text {cor.2 }}$ in a solution of $0.1 \mathrm{M} \mathrm{Na}_{2} \mathrm{SO}_{4}+\mathrm{H}_{2} \mathrm{SO}_{4}, \mathrm{pH} 2$ is shifted by approximately $0.06 \mathrm{~V}$ in the direction of negative potential, in relation to the corrosion potential, in a solution with a $\mathrm{pH}$ of 1 . This might be expected taking into account the influence of $\mathrm{pH}$ on position of the cathodic Tafel lines. Position of the anodic Tafel line does not depend on the $\mathrm{pH}$ value of the solution. Also, for the same reason, the corrosion current in the solution with $\mathrm{pH}$ 2 has a lower value than in a solution with $\mathrm{pH} 1$.
On the oxide surface of passive chromium (Figure 5) hydrogen also evolves by the Volmer-Heyrovsky mechanism [3]:

$$
\mathrm{Cr}_{\mathrm{ox}}+2 \mathrm{H}^{+}+2 \mathrm{e}^{-} \rightarrow \mathrm{Cr}_{\mathrm{ox}}+\mathrm{H}_{2}
$$

At higher cathodic polarization of the passive chromium, at a $\mathrm{pH}>3$, the limiting diffusion current of $\mathrm{H}^{+}$ions can be seen, while at still higher polarizations Tafel lines independent on the $\mathrm{pH}$ value appear, that are characteristic for discharging of $\mathrm{H}_{2} \mathrm{O}$ molecules [3]:

$$
\mathrm{Cr}_{\mathrm{ox}}+2 \mathrm{H}_{2} \mathrm{O}+2 \mathrm{e}^{-} \rightarrow \mathrm{Cr}_{\mathrm{ox}}+\mathrm{H}_{2}+2 \mathrm{OH}^{-}
$$




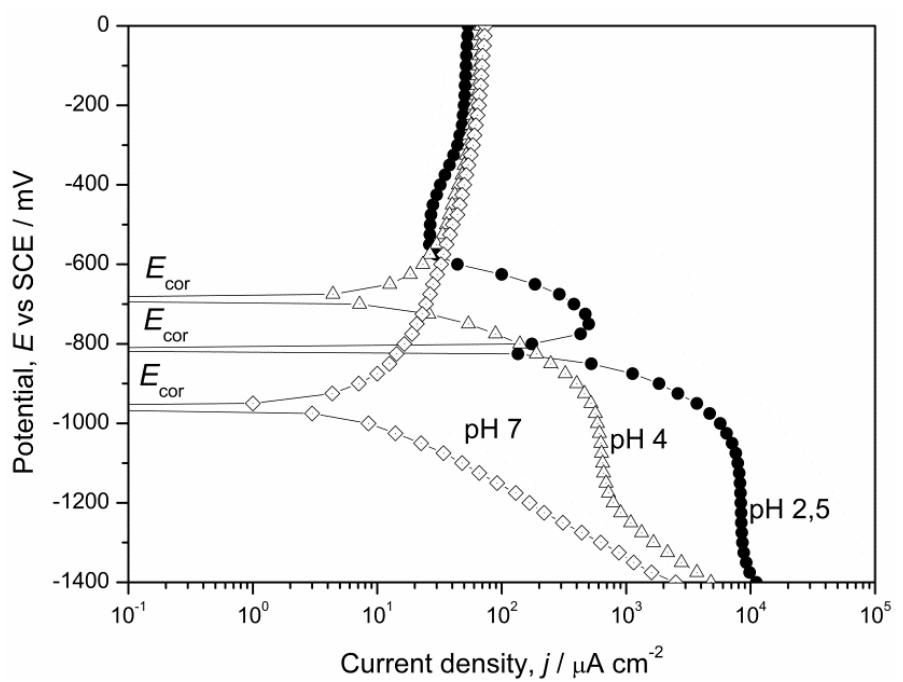

Figure 5 Anodic and cathodic polarization curves for the electrode of chromium, depending on the $\mathrm{pH}$ value of the solution.

In solutions of $0.1 \mathrm{M} \mathrm{Na}_{2} \mathrm{SO}_{4}+\mathrm{H}_{2} \mathrm{SO}_{4}$, $\mathrm{pH}<3$ hydrogen evolves depending on experimental conditions, according to the following three different mechanisms:

1. Electrochemical evolution of $\mathrm{H}_{2}$ by reaction of $\mathrm{H}^{+}$ions according to the Volmer-Heyrovsky mechanism, on the bare chromium surface,

2. During chemical dissolution of chromium by the Kolotyrkin mechanism [2], which does not depend on the electrode potential, and

3. Electrochemical hydrogen evolution as under (1) but on the oxide covered chromium surface (ie, on the passive chromium).

At $\mathrm{pH}>3$ the fourth mechanism of electrochemical hydrogen evolution is observed, that is reaction of water molecules on the oxide covered chromium surface.

\section{CONCLUSION}

Corrosion potential which is formed on the surface of passivated chromium $\left(E_{\text {cor.1 }}\right)$ is due to the cathodic reaction of hydrogen evolution on the oxide covered chromium surface, and the anodic reaction of chromium dissolution through the passive film. Corrosion potential which is formed on the surface of bare chromium is the result of cathodic hydrogen evolution and anodic dissolution of chromium from the bare surface. In this case, the established corro-sion potential is $E_{\text {cor.2. }}$.

In sulfate solutions in the $\mathrm{pH}<3$ range, hydrogen is evolved according to three different mechanisms, depending on the experimental conditions: (1) the electrochemical evolution of $\mathrm{H}_{2}$ by reaction of $\mathrm{H}^{+}$ ions according to the Volmer-Heyrovsky mechanism on the bare chromium surface, (2) during chemical dissolution of chromium by the Kolotyrkin mechanism, which does not depend on electrode potential, and (3) electrochemical hydrogen evolution by the Volmer-Heyrovsky mechanism on the oxide covered chromium surface. At $\mathrm{pH}>3$, the fourth mechanism of hydrogen evolution is observed (4), that is electrochemical reaction of water molecules on the oxide covered chromium surface. 


\section{REFERENCES}

[1] Wilde B. E., Hodge F. G., The Cathodic Discharge of Hydrogen on Active and Passive Chromium Surfaces in Dilute Sulphuric Acid Solutions, Electrochim. Acta 14 (7) (1969) pp. 619627;

[2] Kolotyrkin Ya. M., Florianovich G.M., Anomalnoe rastvorenie metallov. Eksperimentalnie fakti i ih teoreticheskoe tolkovanie, Zashch. Metal. 20 (1) (1984) pp. 14-24;

[3] Dražić D.M., Popić J.P., Anomalous Dissolution of Metals and Chemical Corrosion (Review), J. Serb. Chem. Soc. 70 (3) (2005) pp. 489-513;

[4] Sukhotin A. M., Khoreva N. K., Passivnost khroma. Osobenosti katodnogo aktivirovaniya khroma, Electrokhimiya 18 (1) (1982) pp. 132-134;

[5] Jegdić B., Dražić D. M., Popić J. P., Structural Effects of Metallic Chromium on its Electrochemical Behavior,
J. Serb. Chem. Soc. 72 (6) (2007) pp. 563-578;

[6] Jegdić B., Dražić D. M., Popić J. P., Influence of Chloride Ions on the Open Circuit Potentials of Chromium in Deaerated Sulfuric Acid Solutions, J. Serb. Chem. Soc. 71 (11) (2006) pp. 1187-1194.

[7] Jegdić B., Dražić D. M., Popić J. P., Open Circuit Potentials of Metallic Chromium and Austenitic 304 Stainless Steel in Aqueous Sulphuric Acid Solution and the Influence of Chloride Ions on them, Corr. Sci. 50 (2008) pp. 1235-1244;

[8] Jegdić B., Stevanović M., Jegdić A., Chemical and Electrochemical Dissolution of Chromium at Room and Elevated Temperatures, Mining and Metallurgy Engineering Bor (accepted paper). 


\begin{tabular}{ll}
\hline \hline INSTITUT ZA RUDARSTVO I METALURGIJU BOR & ISSN: 2334-8836 (Štampano izdanje) \\
UDK: 622 & ISSN: 2406-1395 (Online) \\
\hline \hline
\end{tabular}

UDK: $620.193: 546.76 / .11(045)=163.41$

doi:10.5937/MMEB1503145J

Bore Jegdić, Biljana Bobić, Aleksandar Jegdić, Maja Stevanovic ${ }^{*}$

MEHANIZMI IZDVAJANJA VODONIKA NA HROMU***

Izvod

U ovom radu analizirani su mehanizmi izdvajanja vodonika na metalnom hromu u rastvoru $0,1 \mathrm{M}$ $\mathrm{Na}_{2} \mathrm{SO}_{4}+\mathrm{H}_{2} \mathrm{SO}_{4}$, pH 1 do $\mathrm{pH} 7$. U oblasti $\mathrm{pH}<3$ vodonik se izdvaja u zavisnosti od eksperimentalnih uslova po tri različita mehanizma: elektrohemijskim izdvajanjem $\mathrm{H}_{2}$ reagovanjem $\mathrm{H}^{+}$jona po mehanizmu Folmer-Hejrovski na čistoj površini hroma, pri hemijskom rastvaranju hroma po mehanizmu Kolotirkina, a koje ne zavisi od elektrodnog potencijala i elektrohemijskim izdvajanjem vodonika po mehanizmu Folmer-Hejrovski, ali na pasivnom hromu. Pri pH vrednostima većim od 3 uočava se $i$ četvrti mehanizam izdvajanja vodonika elektrohemijskim reagovanjem molekula vode na oksidom presvučenoj površini hroma.

Ključne reči: hrom, korozija, hemijsko rastvaranje, izdvajanje vodonika

\section{UVOD}

Paralelno sa elektrohemijskim rastvaranjem hroma odvija i njegovo hemijsko rastvaranje, koje ne zavisi od potencijala i koje je u nekim uslovima dominantan proces rastvaranja [1-8]. Hemijsko rastvaranje hroma je uzrok pojavi izdvajanja vodonika, koja ne podleže zakonitostima elektrohemijske kinetike. Pojava hemijskog izdvajanja vodonika može biti objašnjenje za uočeno odvijanje procesa korozionog zamora i naponske korozije nerđajućih čelika u uslovima kada je nemoguće njegovo elektrohemijsko izdvajanje. Nisko iskorišćenje struje pri nanošenju prevlake hroma, može biti posledica paralelnog hemijskog rastvaranja hroma tokom galvanskog taloženja hroma.

Hrom se nalazi u pasivnom stanju posle uranjanja u rastvor za ispitivanje, sa karakterističnim korozionim potencijalom $E_{\mathrm{kor}, 1}$.
Katodnom polarizacijom na $-0,900 \mathrm{~V} \mathrm{u}$ toku nekoliko desetina sekundi, pasivni film se rastvara, pri čemu se elektroda aktivira. Tada se formira korozioni potencijal $E_{\mathrm{kor}, 2}$ koji odgovara aktivnom hromu.

\section{EKSPERIMENTALNI DEO}

Uzorci metalnog hroma su zatopljeni u metakrilatnu masu, površina uzoraka je mehanički polirana brusnim papirom do finoće 1000 . Uzorci su zatim ispirani destilovanom vodom i stavljeni u elektrolitičku ćeliju radi ispitivanja. Rastvori za ispitivanje bili su $0,1 \mathrm{M} \mathrm{Na}_{2} \mathrm{SO}_{4}+\mathrm{H}_{2} \mathrm{SO}_{4}, \mathrm{pH} 1$ do $\mathrm{pH}$ 7 , koji su deaerisani provođenjem prečišćenog azota.

Za izvođenje elektrohemijskih ispitivanja korišćena je trodelna staklena elektrohemijska ćelija sa vodenim plaštom za

\footnotetext{
*NU Institut za hemiju, tehnologiju i metalurgiju, Univerziteta u Beogradu, Njegoševa 12, Beograd, Srbija,e-mail: borejegdic@yahoo.com

** Ovaj red je finansiran od strane Ministarstva prosvete, nauke i tehnološkog razvoja Republike Srbije, Projekti No. 34028 i No. 34016.
} 
termostatiranje, sa Pt pomoćnom elektrodom i zasićenom kalomelovom elektrodom (ZKE) kao referentnom elektrodom. Svi izmereni potencijali prikazani su u odnosu na ZKE.

U nekim slučajevima, pre merenja, elektroda hroma je aktivirana katodnom polarizacijom na $-0,900 \mathrm{~V}$ u toku $120 \mathrm{~s}$, radi uklanjanja površinskog oksida, koji se na hromu spontano stvara u dodiru sa vazduhom. Posle $\sim 15$ min stabilizacije korozionog potencijala polarizacione krive su snimane potenciodinamički, pri linearnoj brzini promene potencijala od $2 \mathrm{mV} \mathrm{s}^{-1}$. Elektrohemijska merenja su izvedena primenom potenciostata-galvanostata PAR 273.
Preferencijalna orijentacija pojedinih zrna na površini uzorka hroma (tekstura uzoraka) je određena metodom difrakcije odbijenih elektrona (electron backscatter diffraction, EBSD).

\section{REZULTATI I DISKUSIJA}

Na slici 1 je prikazana inverzna polarna slika teksture uzorka hroma dobijena EBSD metodom. Kao što se vidi sa obojene skale, relativna učestalost kristalnih ravni sa orijentacijom (111) na površini uzorka je 4,851 puta veća $u$ odnosu na učestalost te orijentacije na uzorku bez teksture.

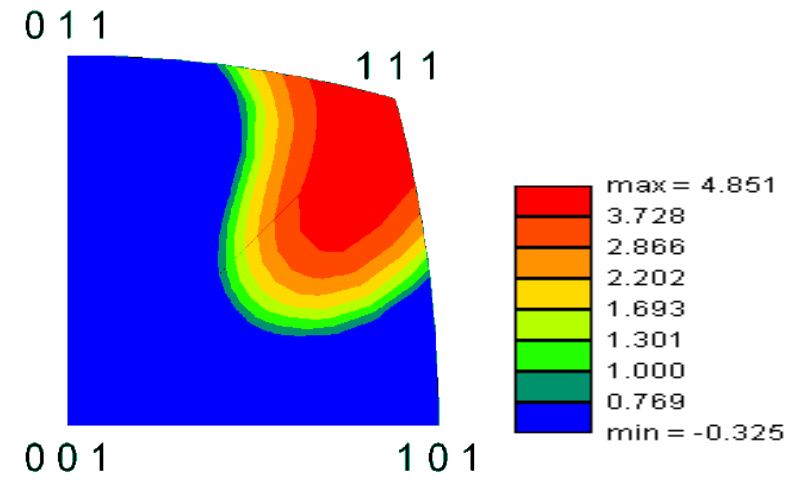

S1. 1. Inverzna polarna slika teksture uzorka hroma dobijena EBSD metodom. Obojena skala označava relativnu učestalost pojedinih kristalografskih orijentacija

Na slici 2 je prikazan ciklični voltamogram za elektrodu hroma u rastvoru $0,1 \mathrm{M}$ $\mathrm{Na}_{2} \mathrm{SO}_{4}+\mathrm{H}_{2} \mathrm{SO}_{4}$, pH 1. Ciklični voltamogram pokazuje neobično ponašanje pri reversnoj promeni potencijala. Naime, registrovana struja umesto da bude uvek anodna $\mathrm{u}$ oblasti potencijala između korozionog potencijala $E_{\mathrm{kor}, 2}$ i pozitivne granice promene potencijala (isečak na slici 2), menja svoj znak dva puta u toj oblasti potencijala, formirajući još jedan korozioni potencijal $E_{\mathrm{kor}, 1}$ i katodni pik, koji nije pik redukcije oksida. Da se radi o katodnom piku izdvajanja vodonika na pasivnom filmu, a ne o redukciji oksida, utvrđeno je potencio- statskim ispitivanjem $\mathrm{u}$ toj oblasti potencijala. Tokom potenciostatskog zadržavanja potencijala u navedenoj oblasti, katodna struja je imala stabilnu vrednost, koja se nije menjala tokom vremena, što ukazuje da se ne radi o redukciji oksida.

Korozioni potencijal $E_{\text {kor.1 }}$, se obrazuje na elektrodi hroma neposredno uronjenoj $\mathrm{u}$ rastvor (koja ima spontano formiran oksidni sloj na svojoj površini), a $E_{\mathrm{kor} .2}$ se odnosi na korozioni potencijal koji se obrazuje na površini hroma posle katodnog tretmana na potencijalu $-0,900 \mathrm{~V} \quad \mathrm{u}$ cilju redukcije prisutnog oksidnog filma. 


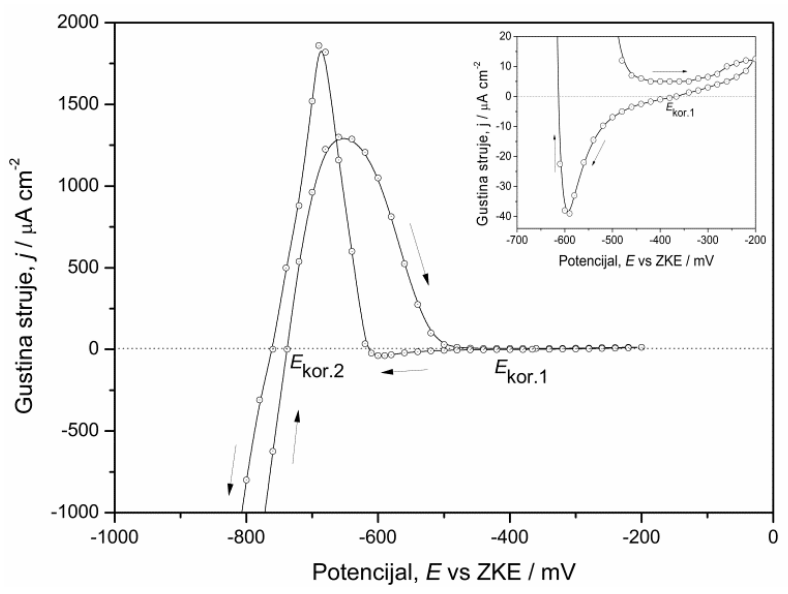

S1. 2. Ciklični voltamogram za elektrodu hroma posle katodne aktivacije. Isečak prikazuje uvećano katodni pik pri povratnoj promeni potencijala koji predstavlja katodno izdvajanje vodonika na pasiviranoj površini.

Primer voltamograma, sličnog onom na isečku na slici 2, je prikazan na slici 3. Reversni delovi cikličnih voltamograma, snimljeni na različitim temperaturama, predstavljaju katodno izdvajanje vodonika na pasiviranoj površini hroma. Kao što se može videti, katodna struja se povećava sa povećanjem temperature, međutim položaj korozionog potencijala $E_{\text {kor, } 1}$ i katodnog pika se ne menja.
Prema tome, katodne struje su posledica katodnog izdvajanja vodonika na hromu prekrivenom oksidom, a korozioni potencijal $E_{\mathrm{kor}, 1}$ je kontrolisan tom reakcijom katodnog izdvajanja vodonika i reakcijom anodnog rastvaranja hroma kroz pasivni film. Vodonik se izdvaja na pasiviranom hromu sve do dostizanja potencijala koji odgovara potencijalu rastvaranja oksida $(\sim-0,580 \mathrm{~V})$. Uzorak hroma se zatim aktivira, a struja naglo prelazi iz katodne $\mathrm{u}$ anodnu oblast.

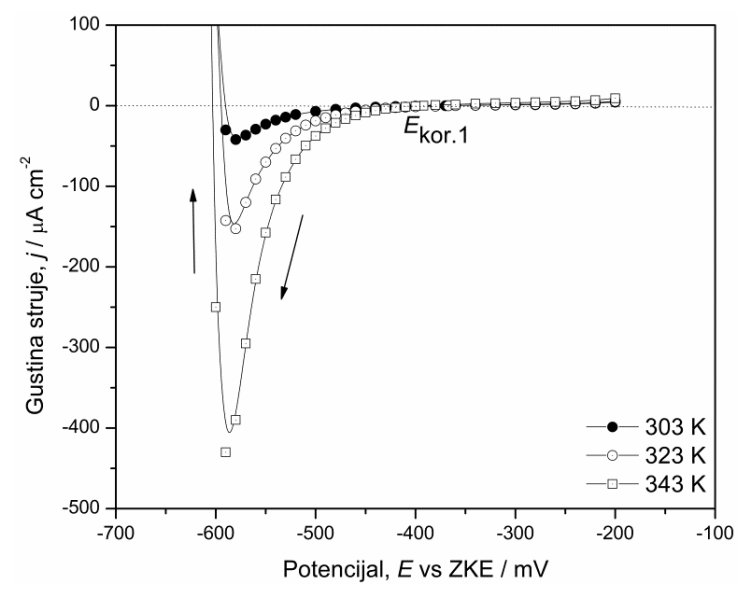

Sl. 3. Reversni delovi voltamograma za elektrodu hroma u rastvoru $0,1 \mathrm{M} \mathrm{Na}_{2} \mathrm{SO}_{4}+\mathrm{H}_{2} \mathrm{SO}_{4}$, pH 1 na različitim temperaturama. 
Na sobnoj temperaturi se Tafelovi nagibi, za elektrodu hroma prekrivenu oksidnim slojem, postepeno menjaju od vrednosti približno jednake $-0,12 \mathrm{~V} \mathrm{dek}^{-1}$ za $\mathrm{pH} 3$ do vrednosti -0,06 $\mathrm{V} \mathrm{dek}^{-1}$ za pH 1 [3].

Polarizacijom hroma prethodno katodno aktiviranog u rastvorima sa $\mathrm{pH}<3$ dobijene su katodne i anodne Tafelove linije sa približno jednakim Tafelovim nagibima od oko $0,12 \mathrm{~V} \operatorname{dek}^{-1}$ (tipični dijagrami za prevlaku $\mathrm{Cr}$ u rastvorima sa $\mathrm{pH} 1 \mathrm{i}$ pH 2 su prikazani na slici 4). Presek Tafelovih linija na korozionom potencijalu $E_{\mathrm{kor} .2}$ pokazuje da je korozioni potencijal određen $\mathrm{u}$ smislu Vagner-Traudovog modela elektrohemijskim procesima anodnog rastvaranja hroma i katodnog izdvajanja vodonika. Paralelno sa elektrohemijskim rastvaranjem odvija se i hemijsko rastvaranje hroma po mehanizmu Kolotirkina [2]. Katodno izdvajanje vodonika na aktivnoj površini hroma sa nagibom od $0,12 \mathrm{~V} \mathrm{dek}^{-1}$ ukazuje na reakciju:

$$
\mathrm{Cr}+2 \mathrm{H}^{+}+2 \mathrm{e}^{-} \rightarrow \mathrm{Cr}+\mathrm{H}_{2}
$$

koja se najverovatnije odvija po mehanizmu Folmer-Hejrovski.

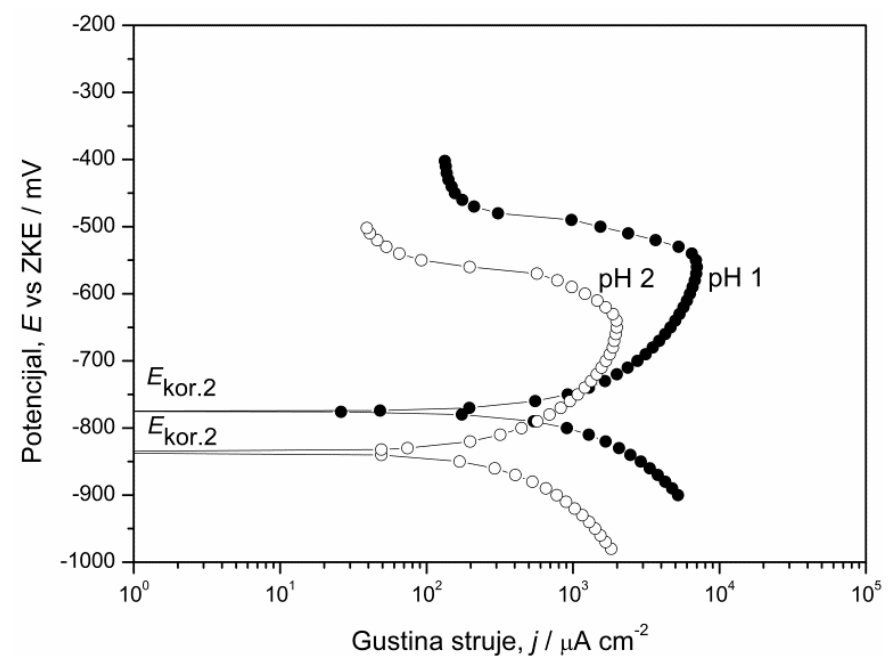

Sl. 4. Anodne i katodne polarizacione krive za elektrodu hroma prethodno katodno aktiviranu, $u$ zavisnosti od $\mathrm{pH}$ vrednosti rastvora

Korozioni potencijal $E_{\mathrm{kor} .2} \mathrm{u}$ rastvoru 0,1 $\mathrm{M} \mathrm{Na} 2 \mathrm{SO}_{4}+\mathrm{H}_{2} \mathrm{SO}_{4}, \mathrm{pH} 2$ je pomeren za približno $0,06 \mathrm{~V}$ u smeru negativnijih potencijala u odnosu na korozioni potencijal u rastvoru sa $\mathrm{pH} 1$, što se moglo i očekivati na osnovu razmatranja uticaja $\mathrm{pH}$ vrednosti na položaj katodnih Tafelovih pravih. Položaj anodne Tafelove prave ne zavisi od $\mathrm{pH}$ vrednosti rastvora. Takođe iz istih razloga struja korozije $\mathrm{u}$ rastvoru sa $\mathrm{pH} 2$ ima nižu vrednost nego u rastvoru sa $\mathrm{pH} 1$.

Na pasivnom hromu (Slika 5) vodonik se takođe izdvaja mehanizmom Folmer-
Hejrovski, ali na površini hroma prekrivenoj oksidnim slojem [3]:

$$
\mathrm{Cr}_{\mathrm{ox}}+2 \mathrm{H}^{+}+2 \mathrm{e}^{-} \rightarrow \mathrm{Cr}_{\mathrm{ox}}+\mathrm{H}_{2}
$$

Pri većim katodnim polarizacijama pasivnog hroma, pri $\mathrm{pH}>3$ uočava se granična difuziona struja $\mathrm{H}^{+}$jona, a pri još većim polarizacijama pojava Tafelovih pravih nezavisnih od $\mathrm{pH}$, karakterističnih za razelektrisanje molekula $\mathrm{H}_{2} \mathrm{O}$ [3]:

$$
\mathrm{Cr}_{\mathrm{ox}}+2 \mathrm{H}_{2} \mathrm{O}+2 \mathrm{e}^{-} \rightarrow \mathrm{Cr}_{\mathrm{ox}}+\mathrm{H}_{2}+2 \mathrm{OH}^{-}
$$




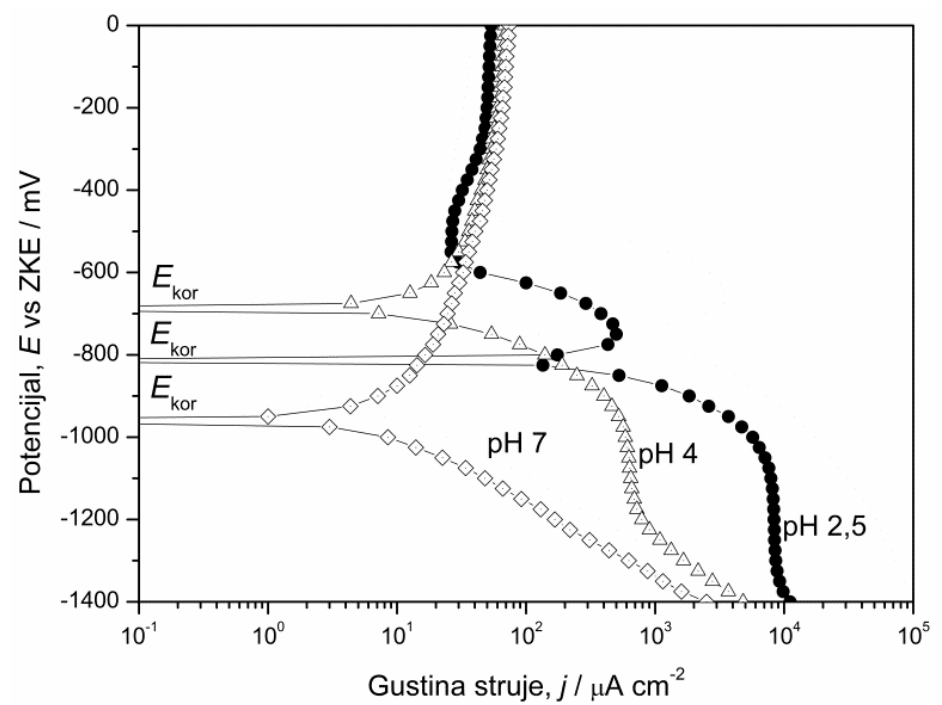

Sl. 5. Anodne i katodne polarizacione krive za elektrodu hroma u zavisnosti od $\mathrm{pH}$ vrednosti rastvora

$\mathrm{U}$ rastvorima $0,1 \mathrm{M} \mathrm{Na} \mathrm{SO}_{4}+\mathrm{H}_{2} \mathrm{SO}_{4}$, $\mathrm{pH}<3$ vodonik se izdvaja $\mathrm{u}$ zavisnosti od eksperimentalnih uslova po tri različita mehanizma:

1. elektrohemijskim izdvajanjem $\mathrm{H}_{2}$ reagovanjem $\mathrm{H}^{+}$jona po mehanizmu Folmer-Hejrovski na čistoj površini hroma,

2. pri hemijskom rastvaranju hroma po mehanizmu Kolotirkina [2], a koje ne zavisi od elektrodnog potencijala, i

3. elektrohemijskim izdvajanjem vodonika kao pod (1) ali na površini hroma prekrivenoj oksidnim slojem.

Pri $\mathrm{pH}$ vrednostima većim od 3 uočava se i četvrti mehanizam izdvajanja vodonika, elektrohemijskim reagovanjem molekula vode na oksidom prevučenj površini hroma.

\section{ZAKLJUČAK}

Korozioni potencijal koji se obrazuje na pasiviranoj površini hroma $\left(E_{\mathrm{kor} .1}\right)$ je posle- dica odvijanja katodna reakcije izdvajanja vodonika i reakcije anodnog rastvaranja hroma kroz pasivni film. Korozioni potencijal koji se obrazuje na čistoj površini hroma je posledica katodnog izdvajanja vodonika i anodnog rastvaranja hroma sa te površine. U tom slučaju uspostavlja se korozioni potencijal $E_{\mathrm{kor} .2}$.

$\mathrm{U}$ sulfatnim rastvorima, u oblasti $\mathrm{pH}<3$ vodonik se izdvaja u zavisnosti od eksperimentalnih uslova po tri različita mehanizma: (1) elektrohemijskim izdvajanjem $\mathrm{H}_{2}$, reagovanjem $\mathrm{H}^{+}$jona po mehanizmu Folmer-Hejrovski na čistoj površini hroma, (2) pri hemijskom rastvaranju hroma po mehanizmu Kolotirkina, a koje ne zavisi od elektrodnog potencijala, i (3) elektrohemijskim izdvajanjem vodonika po mehanizmu Folmer-Hejrovski, ali na površini hroma prekrivenoj oksidnim slojem. Pri $\mathrm{pH}$ vrednostima većim od 3 uočava se i četvrti mehanizam izdvajanja vodonika (4) elektrohemijskim reagovanjem molekula vode na površini hroma prekrivenoj oksidnim slojem. 


\section{LITERATURA}

[1] Wilde B. E., Hodge F. G., The cathodic discharge of hydrogen on active and passive chromium surfaces in dilute sulphuric acid solutions, Electrochim. Acta 14 (7) (1969), str. 619-627.

[2] Kolotyrkin Ya. M., Florianovich G. M., Anomalnoe rastvorenie metallov. Eksperimentalnie fakti i ih teoreticheskoe tolkovanie, Zashch. Metal. 20 (1) (1984), str. 14-24.

[3] Dražić D. M., Popić J. P., Anomalous dissolution of metals and chemical corrosion (Review), J. Serb. Chem. Soc. 70 (3) (2005), str. 489-513.

[4] Sukhotin A. M., Khoreva N. K., Passivnost khroma. Osobenosti katodnogo aktivirovaniya khroma, Electrokhimiya 18 (1) (1982), str. 132-134.

[5] Jegdić B., Dražić D. M., Popić J. P., Structural effects of metallic chromium on its electrochemical behavior, J.

Serb. Chem. Soc. 72 (6) (2007), str. 563-578.

[6] Jegdić B., Dražić D. M., Popić J. P., Influence of chloride ions on the open circuit potentials of chromium in deaerated sulfuric acid solutions, J. Serb. Chem. Soc. 71 (11) (2006), str. 11871194.

[7] Jegdić B., Dražić D.M., Popić J.P., Open circuit potentials of metallic chromium and austenitic 304 stainless steel in aqueous sulphuric acid solution and the influence of chloride ions on them, Corr. Sci. 50 (2008), str. 12351244.

[8] Jegdić B., Stevanović M., Jegdić A., Chemical and Electrochemical Dissolution of Chromium at Room and Elevates Temperatures, Mining and Metallurgy Engineering Bor (accepted paper). 